\title{
ICTIOFAUNA DO RIO JAGUARIBE, UMA MICROBACIA SOB DOMÍNIO DA MATA ATLÂNTICA, PARÁíBA, NORDESTE DO BRASIL
}

\author{
ICHTHYOFAUNA OF THE JAGUARIBE RIVER, A MICROBASIN UNDER \\ THE INFLUENCE OF THE MATA ATLÂNTICA, PARAÍBA, NORTHEAST BRAZIL
}

\author{
Telton Pedro Anselmo Ramos ${ }^{1 \star}$ \\ Yuri Gomes Ponce de Carvalho Rocha ${ }^{2}$ \\ Antônio Augusto de Sá Neto ${ }^{3}$ \\ Márcio Joaquim da Silva ${ }^{4}$ \\ Gildo Gomes Filho ${ }^{5}$ \\ Ricardo de Souza Rosa ${ }^{6}$
}

\section{Resumo}

O inventário da ictiofauna do rio Jaguaribe, pequena bacia costeira localizada dentro do perímetro urbano da grande João Pessoa, Estado da Paraíba revelou um total de 21 espécies de peixes, distribuídas em 18 gêneros, 11 famílias e sete ordens. Deste total, 17 espécies são de água doce e cinco marinho-estuarinas. As ordens mais especiosas foram Characiformes com nove espécies ( $42,8 \%$ da amostragem), todas dulcícolas, e Perciformes com seis espécies $(28,5 \%)$, das quais três são dulcícolas e quatro marinho-estuarinas. Characidae foi a família com maior número de espécies (6 spp., 28,5\%), seguida de Cichlidae (3 spp., 14,0\%). Duas das espécies de água doce não são nativas, a tilápia (Oreochromis niloticus) e o barrigudinho (Poecilia reticulata). Das 15 espécies de água doce nativas, nove (60\%) foram registradas dentro da área da Unidade de Conservação Refúgio da Vida Silvestre Mata do Buraquinho, a qual é cortada pelo curso principal do rio Jaguaribe. A riqueza de espécies registrada neste estudo (21) mostra-se similar ao que tem sido registrado em outros rios costeiros sobre influência da Mata Atlântica da ecorregião Nordeste Médio-Oriental.

1 Universidade Estadual da Paraíba, Laboratório de Ecologia Aquática, Departamento de Biologia/ CCBS, Campus universitário, 58109-753, Campina Grande, PB, Brasil.

*Autor para correspondência: telton@gmail.com

2 Universidade Federal da Paraíba, Centro de Ciências Exatas e da Natureza, Departamento de Sistemática e Ecologia, Laboratório de Ictiologia, Campus I, Jardim Cidade Universitária, CEP 580599oo, João Pessoa, PB, Brasil.

3 Universidade Federal da Paraíba, Centro de Ciências Exatas e da Natureza, Departamento de Sistemática e Ecologia, Laboratório de Ictiologia, Campus I, Jardim Cidade Universitária, CEP 5805990o, João Pessoa, PB, Brasil.

4 Universidade Federal do Pará, Laboratório de Conservação, Ecologia e Sistemática de Peixes, Faculdade de Ciências Biológicas/FCBio, Campus Universitário do Marajó-Soure, 68870-ooo, Soure, PA, Brasil.

5 Universidade Federal da Paraíba, Centro de Ciências Exatas e da Natureza, Departamento de Sistemática e Ecologia, Laboratório de Ictiologia, Campus I, Jardim Cidade Universitária, CEP 580599oo, João Pessoa, PB, Brasil.

6 Universidade Federal da Paraíba, Centro de Ciências Exatas e da Natureza, Departamento de Sistemática e Ecologia, Laboratório de Ictiologia, Campus I, Jardim Cidade Universitária, CEP 580599oo, João Pessoa, PB, Brasil. 
Palavras-chave: conservação, inventário, peixes, rios costeiros, sistemática.

\begin{abstract}
The inventory of the ichthyofauna of the Jaguaribe River, a small coastal basin located within the urban perimeter of João Pessoa, State of Paraíba, revealed a total of 21 fish species, distributed in 18 genera, 11 families and seven orders. Of this total, 17 species are freshwater and five of marine-estuarine origin. The most speciose order is Characiformes with nine species (42.8\% of the sample), all freshwater, followed by Perciformes with six species (28.5\%), of which three are freshwater and four are estuarine. Characidae was the family with the highest number of species (six, 28.5\%), followed by Cichlidae (three, 14.0\%). Two of the freshwater species are introduced, Oreochromis niloticus and Poecilia reticulata. Of the 15 freshwater species, nine $(60 \%)$ were recorded within the protected area of the Mata do Buraquinho Wildlife Refuge, which is drained by the main course of the Jaguaribe River. The species richness recorded in this study (21) is similar to those of other coastal rivers under the influence of the Atlantic Forest of the Northeastern Middle East ecoregion.
\end{abstract}

Keywords: conservation, inventory, fishes, coastal rivers, systematics

\title{
Resumen
}

El inventario de la ictiofauna del río Jaguaribe, una pequeña cuenca costera ubicada dentro del perímetro urbano de João Pessoa, estado de Paraíba, reveló un total de 21 especies de peces, distribuidas en 18 géneros, 11 familias y siete órdenes. De este total, 17 especies son de agua dulce y 5 de origen marino-estuarinas. Los órdenes con mayor número de especies fueron Characiformes con nueve especies (42,8\% del total), todas de agua dulce, seguido de Perciformes con seis especies $(28,5 \%)$, de las cuales tres son de agua dulce y cuatro son estuarinas. Characidae fue la familia con mayor número de especies (6 spp; 28,5\%), seguida de Cichlidae (3 spp; 14,0\%). Dos de las especies de agua dulce son introducidas, la tilapia Oreochromis niloticus y el "barrigudinho" Poecilia reticulata. De las 15 especies nativas de agua dulce, nueve (6o\%) fueron registradas dentro del área de la Unidad de Conservación del Refugio de Vida Silvestre Mata do Buraquinho, la cual es atravesada por el cauce principal del río Jaguaribe. La riqueza de especies registrada en este estudio (21) es similar a la registrada en otros ríos costeros bajo la influencia de la Mata Atlántica de la ecorregión Nordeste Medio-Oriental.

Palabras clave: conservación, inventario, peces, ríos costeros, sistemática

\section{INTRODUÇÃO}

O Brasil detém substancial parcela da ictiofauna de água doce Neotropical, sendo o país com a maior diversidade deste grupo do mundo. Somava, na década 
passada, 2.587 espécies válidas de famílias que ocorrem estritamente em sistemas dulcícolas (Buckup et al. 2007). Atualmente, são reconhecidas em torno de 3.500 espécies de peixes de água doce no Brasil, das quais cerca de 1.800 são endêmicas (Reis et al. 2016). No entanto, até pouco tempo, o conhecimento desta diversidade não era uniformemente distribuído no território nacional, sendo a ictiofauna dulcícola da região Nordeste do Brasil uma das menos conhecidas (Langeani et al. 2009). Nas últimas décadas, este quadro vem mudando, com recentes trabalhos publicados sobre a diversidade de peixes de água doce desta região (Ramos et al. 2005; Burger et al. 2011; Camelier e Zanata 2014; Ramos et al. 2014; Paiva et al. 2014; Silva et al. 2014; Silva et al. 2015; Lima et al. 2017; Ramos et al. 2018) assim como novas espécies descritas (Vari et al. 2010; Zanata e Serra 2010; Zanata et al. 2013; Costa et al. 2010; Ramos et al. 2013;) e novos registros (Sarmento-Soares et al. 2016).

Paiva (1995) afirmou que a fauna de peixes é bastante depauperada nas bacias hidrográficas situadas entre os rios Parnaíba e São Francisco, compreendendo apenas cerca de 50 espécies e indica as condições ambientais adversas como principal causa da baixa diversidade. Entretanto, Rosa et al. (2003) registraram 82 espécies nas mesmas bacias e chamaram, pela primeira vez, esta região hidrográfica de Nordeste Médio-Oriental. Mais recentemente, Abell et al. (2008) denominaram esta área de Ecorregião Hidrográfica Northeastern Caatinga and Coastal Drainages e estimaram sua riqueza de peixes entre 67 e 101 espécies. Posteriormente, Lima et. al (2017) listaram 103 espécies nestas bacias e a nomearam de "Mid-Northeastern Caatinga ecoregion".

Poucos são os trabalhos de levantamento ictiofaunístico em rios costeiros da região Nordeste, como a bacia do rio Jaguaribe, foco deste estudo. Torelli et al. (1997) e Gomes-Filho e Rosa (2001) realizaram inventários da ictiofauna do rio Gramame, Estado da Paraíba. Gomes-Filho (1999) fez um levantamento das espécies de peixes da ordem Characiformes nas bacias costeiras do Estado da Paraíba e apontou a necessidade de estudos adicionais, devido ao baixo grau de amostragem desses rios costeiros. Paiva et al. (2014) estudaram a ictiofauna da microbacia do rio Pratagi, localizada na Mata Atlântica do Estado do Rio Grande do Norte.

Os peixes de Mata Atlântica são aqueles associados aos cursos d'água que drenam as regiões da Floresta Ombrófila Densa (Buckup 1998). Este autor, indicou as bacias costeiras do litoral de Pernambuco, localizadas a leste do Planalto da Borborema, como o segmento setentrional deste bioma. Em função da falta de inventários completos sobre a ictiofauna das bacias hidrográficas da Mata Atlântica, pouco se sabe sobre sua riqueza de espécies. No entanto, acredita-se que o grau de endemismo seja elevado (Buckup 1998; Menezes et al. 2007). Portanto, estudos de levantamento da fauna de peixes são necessários para melhorar a informação sistemática sobre este grupo na Mata Atlântica, além de identificar quais espécies são possivelmente endêmicas deste bioma.

No campo da ecologia da conservação, a maioria dos peixes brasileiros reconhecidos como ameaçados de extinção são espécies de água doce. Nas áreas de Mata Atlântica a degradação ambiental provocada por desmatamento, ocupação 
humana descontrolada e poluição, contribuiu decisivamente na diminuição de estoques e populações de peixes, redução da diversidade original e, em alguns casos, possivelmente no desaparecimento de espécies antes mesmo de suas descrições (Menezes et al. 2007). A destruição da Mata Atlântica é considerada como um dos principais fatores na eliminação de espécies de peixes de água doce brasileiros (Buckup 1998). A inexistência de publicações tratando do inventário ou dos aspectos biológicos da ictiofauna do rio Jaguaribe, acrescido ao fato de que o rio está visivelmente comprometido pela poluição hídrica, retirada da mata ciliar e canalização, reforça a importância deste levantamento, buscando embasar ações de avaliação de impactos, recuperação ambiental e conservação e manejo ictiofauna da Mata Atlântica.

\section{MATERIAL E MÉTODOS}

\section{Área de estudo}

O rio Jaguaribe está localizado na Mesorregião da Mata Paraibana, entre as coordenadas $-7.05582^{\circ}$ a $-7.16836^{\circ} \mathrm{S}$ e $-34.84316^{\circ}$ a $-34.90298^{\circ} \mathrm{W}$ (Figura 1 ). Seu curso principal possui $21 \mathrm{~km}$ de extensão e sua bacia de drenagem ocupa uma área de 11,6 $\mathrm{km}^{2}$. Apresenta como limites a bacia do rio Paraíba do Norte, ao Norte; o rio Cuiá, ao Sul; o Oceano Atlântico, a Leste e as bacias dos rios Marés, Cabelo, Jacarapé e Aratu, a Oeste (Melo et al. 2001).

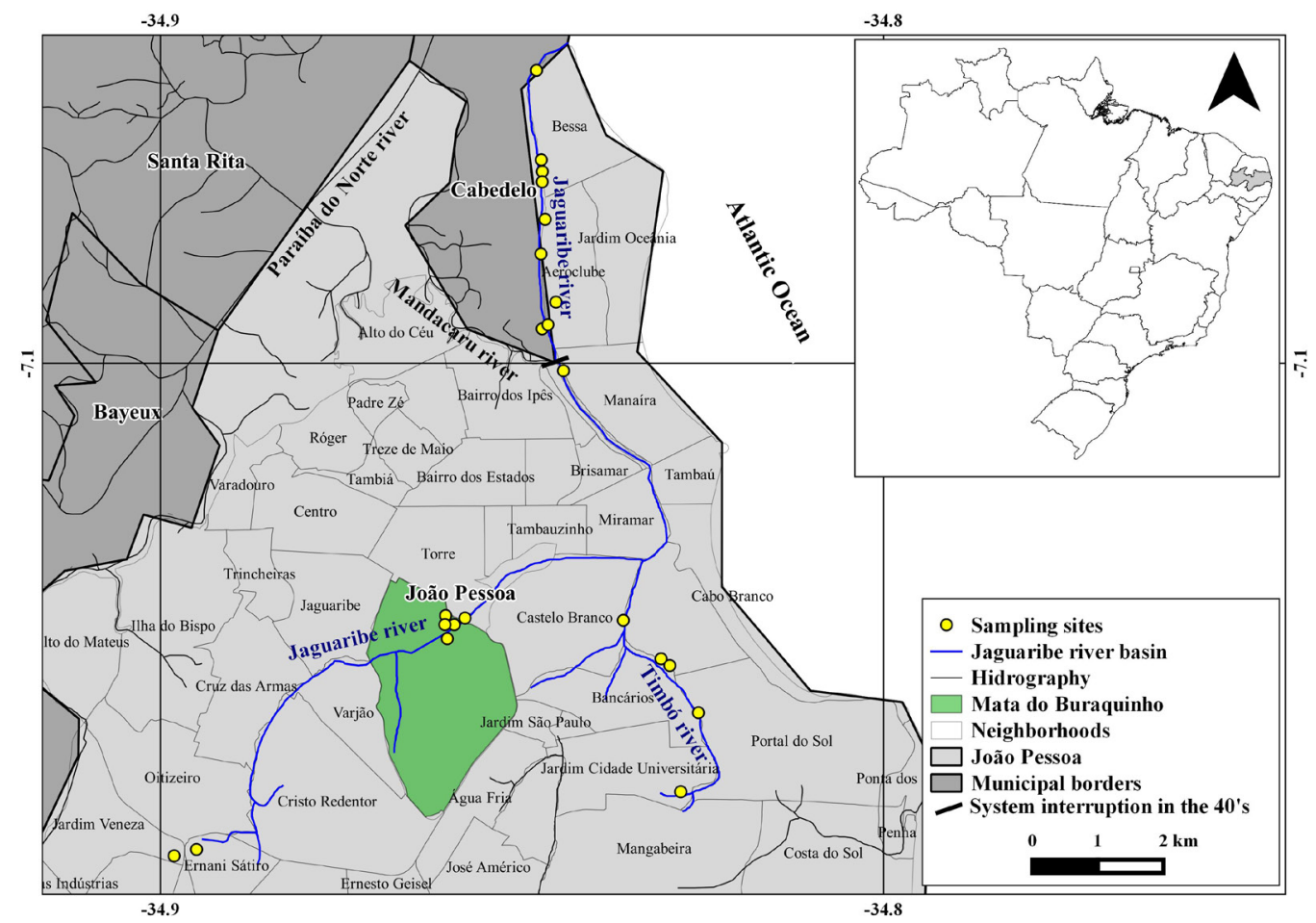

Figura 1. Mapa da bacia do rio Jaguaribe, Estado da Paraíba, Brasil, indicando os pontos de amostragem. 
A nascente do rio Jaguaribe localiza-se na Zona Sul do município de João Pessoa, nas imediações dos bairros Ernani Sátiro e Jardim Veneza, aproximadamente na cota topográfica de 4om, sobre a formação do macro-relevo, denominada de Baixos Planaltos Costeiros. Seu curso prossegue no sentido João Pessoa-Cabedelo, cruzando a Mata do Buraquinho (Figura 1). Essa mata possui em torno de 519,75 hectares, sendo considerada a área de Mata Atlântica mais representativa do Estado da Paraíba (Barbosa 1996). Na década de 1920, o governo do Estado alterou o curso principal da porção baixa do rio Jaguaribe com a construção de um canal, ligando-o ao rio Mandacaru, afluente do rio Paraíba do Norte (Rosa 2009). Em 2014, sua importância para a conservação das espécies que ali vivem foi reconhecida através da publicação do decreto estadual 35.195/2014. Esse documento constituiu essa floresta como Unidade de Conservação (UC) da categoria de Refúgio da Vida Silvestre, passando a ser denominada Jardim Botânico Benjamim Maranhão.

O rio Jaguaribe tem como principal afluente o rio Timbó, que nasce na divisa dos bairros Jardim Cidade Universitária e Mangabeira e segue seu rumo em direção Norte (aproximadamente cinco quilômetros) até a confluência com a margem direita do rio principal, entre os bairros do Altiplano Cabo Branco, Miramar e Castelo Branco (Figura 2) (Melo et al. 2001).
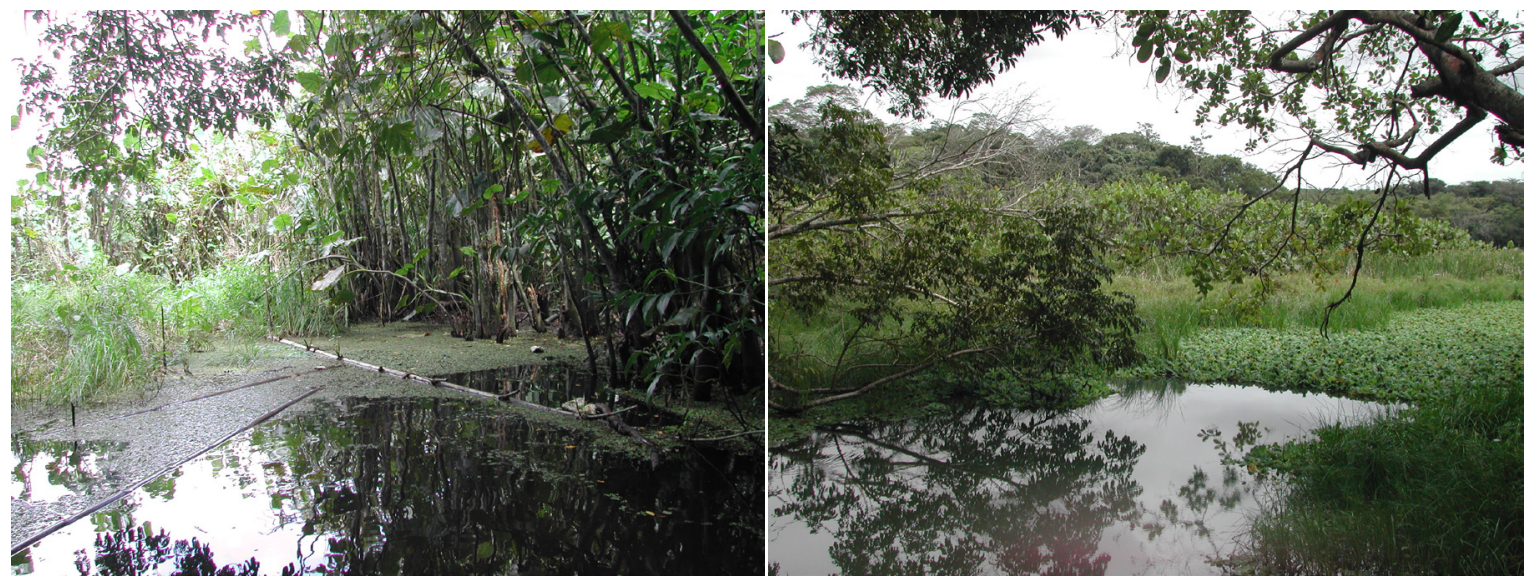

Figura 2. Curso do rio Jaguaribe, na Unidade de Conservação Refúgio da Vida Silvestre Jardim Botânico Benjamim Maranhão.

\section{Coleta de dados}

O material de estudo é proveniente da Coleção Ictiológica da Universidade Federal da Paraíba (UFPB), complementado com coletas adicionais realizadas nos anos de 2017 e 2018. Os peixes foram coletados com redes de arrasto e emalhe, tarrafa, peneiras e puçás durante várias expedições realizadas no rio Jaguaribe e no rio Timbó. Portanto, os dados não obedecem a uma única metodologia de coleta e aqui são apresentados como dados qualitativos. 
Os espécimes foram fixados em solução de formol 10\% e transferidos para frascos de vidro contendo álcool a 70ㅜㅡ (Malabarba e Reis 1987). Após a fixação, os peixes foram triados e identificados no Laboratório de Ictiologia da UFPB com auxílio de lupas estereoscópicas. A identificação sistemática do material baseou-se em Gomes-Filho (1999) para os Characiformes, Reis (1993) para os Callichthyidae, Ihering (1931) para os Poeciliidae, Kullander (1983) para o gênero Cichlasoma, Gosse (1975) para o gênero Geophagus e Britski et al. (1984) e Ramos et al. (2018) para os demais grupos taxonômicos. Dúvidas persistentes foram discutidas com especialistas em cada grupo taxonômico.

A classificação sistemática seguiu Fricke et al. (2019) e o endemismo foi avaliado para a bacia estudada, em consulta aos trabalhos de e Buckup et al. (2007). A presença de espécies ameaçadas foi checada através de consulta a "Lista de espécies de peixes e invertebrados aquáticos ameaçados de extinção do Brasil (Brasil, 2014). Além disso, o material examinado foi listado em ordem alfabética de gêneros no material suplementar do presente estudo (Anexo 1).

\section{RESULTADOS}

$\mathrm{Na}$ bacia do rio Jaguaribe foram registradas 21 espécies de peixes, distribuídas em 18 gêneros, 11 famílias e sete ordens. Deste total, 18 espécies são de água doce e quatro marinho-estuarinas (Tabela 1). Duas das espécies de água doce não são nativas, a tilápia, Oreochromis niloticus (Linnaeus, 1758), o barrigudinho, Poecilia reticulata Peters, 1860 . Das 15 espécies de água doce nativas, nove (6o\%) foram registradas dentro da área da Unidade de Conservação Refúgio da Vida Silvestre Mata do Buraquinho - REVIS (Tabela 1). A espécie Hemigrammus cf. unilineatus (Gill, 1858) foi a única espécie registrada apenas dentro da REVIS. Steindachnerina notonota (MirandaRibeiro 1937), Astyanax aff. fasciatus (Cuvier 1819), Hemigrammus marginatus Ellis 1911, Hyphessobrycon parvellus Ellis 1911, Megalechis thoracata (Valenciennes 1840) e Gymnotus aff. carapo Linnaeus, 1758 foram coletados exclusivamente fora da Unidade de Conservação. Das espécies marinho-estuarinas listadas, apenas Dormitator maculatus (Bloch,1792) foi registrada na área de abrangência da Mata do Buraquinho. 
Tabela 1. Lista sistemática da ictiofauna da bacia rio Jaguaribe, João Pessoa, Paraíba, Brasil, com os respectivos nomes vulgares. * = Espécies introduzida.

\begin{tabular}{|c|c|c|}
\hline ORDEM/Família/Espécie & HABITAT & VOUCHER \\
\hline \multicolumn{3}{|l|}{ CHARACIFORMES (9) } \\
\hline \multicolumn{3}{|l|}{ Curimatidae (1) } \\
\hline Steindachnerina notonota (Miranda-Ribeiro, 1937) & Água doce & UFPB 01383 \\
\hline \multicolumn{3}{|l|}{ Erythrinidae (2) } \\
\hline Hoplias aff. malabaricus (Bloch, 1794) & Água doce & UFPB 05970 \\
\hline Hoplerythrinus unitaeniatus (Agassiz, 1829) & Água doce & UFPB 00350 \\
\hline \multicolumn{3}{|l|}{ Characidae (6) } \\
\hline Astyanax aff. bimaculatus (Linnaeus, 1758) & Água doce & UFPB 00384 \\
\hline Astyanax aff. fasciatus (Cuvier, 1819) & Água doce & UFPB 00475 \\
\hline Compsura heterura Eigenmann, 1915 & Água doce & UFPB 05973 \\
\hline Hemigrammus marginatus Ellis, 1911 & Água doce & UFPB 02168 \\
\hline Hemigrammus unilineatus (Gill, 1858) & Água doce & UFPB 00383 \\
\hline Hyphessobrycon parvellus Ellis, 1911 & Água doce & UFPB 05993 \\
\hline \multicolumn{3}{|l|}{ SILURIFORMES (1) } \\
\hline \multicolumn{3}{|l|}{ Callichthyidae (1) } \\
\hline Megalechis thoracata (Valenciennes, 1840) & Água doce & UFPB 00051 \\
\hline \multicolumn{3}{|l|}{ GYMNOTIFORMES (1) } \\
\hline \multicolumn{3}{|l|}{ Gymnotidae (1) } \\
\hline Gymnotus aff. carapo Linnaeus, 1758 & Água doce & UFPB 00344 \\
\hline \multicolumn{3}{|l|}{ CYPRINODONTIFORMES (2) } \\
\hline \multicolumn{3}{|l|}{ Poecilidae (2) } \\
\hline Poecilia vivipara Bloch \& Schneider, 1801 & Água doce & UFPB 00521 \\
\hline Poecilia reticulata Peters, $1860^{*}$ & Água doce & UFPB 00522 \\
\hline \multicolumn{3}{|l|}{ SYNBRANCHIFORMES (1) } \\
\hline \multicolumn{3}{|l|}{ Synbranchidae (1) } \\
\hline Synbranchus sp. & Água doce & UFPB 00525 \\
\hline \multicolumn{3}{|l|}{ CICHLIFORMES (3) } \\
\hline \multicolumn{3}{|l|}{ Cichlidae (3) } \\
\hline Cichlasoma orientale Kullander, 1983 & Água doce & UFPB 01334 \\
\hline Geophagus brasiliensis (Quoy \& Gaimard, 1824) & Água doce & UFPB 00500 \\
\hline Oreochromis niloticus (Linnaeus, 1758$)^{*}$ & Água doce & UFPB 05978 \\
\hline \multicolumn{3}{|l|}{ GOBIIFORMES ( 2$)$} \\
\hline \multicolumn{3}{|l|}{ Eleotridae (2) } \\
\hline Dormitator maculatus (Bloch,1792) & Estuarino-marinha & UFPB 00003 \\
\hline Eleotris pisonis (Gmelin,1789) & Estuarino-marinha & UFPB oo809 \\
\hline \multicolumn{3}{|l|}{ MUGILIFORMES (1) } \\
\hline \multicolumn{3}{|l|}{ Mugilidae (1) } \\
\hline Mugil curema Valenciennes, 1836 & Estuarino-marinha & UFPB 11582 \\
\hline
\end{tabular}


A ordem mais especiosa foi Characiformes, com nove espécies $(42,8 \%$ da amostragem), todas dulcícolas. Perciformes foi a segunda, com seis $(28,5 \%)$, das quais três são dulcícolas e quatro marinho-estuarinas. Characidae foi a família com maior número de espécies com seis registradas (28.5\%), seguida de Cichlidae com três (14.0\%). Não foram registradas espécies ameaçadas ou endêmicas da ecorregião Nordeste Médio-Oriental.

\section{DISCUSSÃO}

O presente trabalho constitui o primeiro inventário publicado da composição da ictiofauna da bacia do rio Jaguaribe, uma microbacia sob domínio da Mata Atlântica no Estado Paraíba. Os grupos registrados entre os mais representativos [Characiformes (família Characidae) e Perciformes (família Cichlidae)] seguem o padrão verificado em trabalhos de levantamento da ictiofauna de água doce das bacias sob domínio da Mata Atlântica do Nordeste Médio-Oriental (Gomes-Filho 1999; Gomes-Filho e Rosa 2001; Paiva et al. 2014). Além disso, obedece o padrão diagnosticado para todo o Nordeste brasileiro (Rosa et al. 2003; Ramos et al. 2005; Ramos et al. 2014; Silva et al. 2014; Silva et al. 2015), demais áreas do Brasil (Buckup et al. 2007) e da região Neotropical (Reis et al. 2003).

Hemigrammus cf. unilineatus, anteriormente conhecida de outras bacias costeiras da Paraíba, possivelmente faz parte de um grupo de espécies (citado na literatura como Hemigrammus unilineatus), com ocorrência conhecida para Trinidad, bacia do rio Orinoco na Venezuela, bacia do rio Essequibo e rios costeiros do Suriname, rios costeiros da Guiana Francesa, na bacia Amazônica, incluindo o rio Napo no Equador, rios Guaporé e Mamoré na Bolívia, Peru e rio Branco, no Brasil, e no rio Capim no Pará (Reis et al. 2013; Buckup et al. 2007; Ramos et al. 2018). Hemigrammus cf. unilineatus foi registrada em rios costeiros do Nordeste, entre os Estados de Alagoas e Paraíba. Este táxon demanda estudos adicionais para a confirmação de seu estado taxonômico (Géry 1977).

Compsura heterura é uma espécie exclusiva do Nordeste do Brasil eamplamente distribuída na Caatinga das bacias dos rios Parnaíba e São Francisco, e rios costeiros do Nordeste Médio-Oriental incluindo também o bioma da Mata Atlântica. O mesmo padrão de distribuição é observado para as espécies como Hemigrammus marginatus e Serrapinnus heterodon (Rosa et al. 2003; Buckup et al. 2007; Ramos et al. 2014). Outros Characiformes com ampla distribuição no Brasil ou mesmo na América do Sul, como Hoplias malabaricus, Hoplerythrinus unitaeniatus, Astyanax aff. bimaculatus e $A$. fasciatus, podem representar complexos de espécies ainda não resolvidos do ponto de vista taxonômico.

As duas espécies exóticas registradas encontram-se amplamente distribuídas e documentadas na América do Sul: Oreochromis niloticus (Linnaeus, 1758), 
proveniente do continente africano e Poecilia reticulata Peters, 186o, oriunda do Norte da América do Sul (Ramos et al. 2014).

Ressalta-se que na Unidade de Conservação Refúgio da Vida Silvestre Jardim Botânico Benjamim Maranhão foram registradas mais da metade (64,3\%) das espécies de peixes de água doce da bacia do Jaguaribe e um quarto das estuarino-marinhas. Assim como em outros rios costeiros de pequeno porte do bioma Mata Atlântica, destacam-se as ausências de algumas espécies de Characiformes como Apareiodon davisi Fowler 1941 e Psectrogaster saguiru (Fowler 1941) endêmicas da ecorregião Nordeste Médio Oriental e presentes nos rios de médio porte que desaguam no litoral da Paraíba, como os rios Mamanguape e Paraíba do Norte (Gomes-Filho 1999).

Astyanax aff. fasciatus (Cuvier, 1819), conhecida como piaba-do-rabo-vermelho, tinha sua distribuição possivelmente associada ao baixo curso do rio Jaguaribe, em lagoas costeiras e alagados dos bairros de Manaíra, Bessa e Intermares. Um lote da espécie (UFPB 0475) foi coletado em outubro de 1977 nos "Alagados do Bessa, João Pessoa”. Possivelmente, esta espécie desapareceu localmente, uma vez que a área em questão foi amplamente drenada e aterrada para o desenvolvimento urbano. A espécie do gênero Synbranchus Bloch, 1795, popularmente conhecida como mussum, foi tratada como "sp." por não corresponder a Synbranchus marmoratus Bloch, 1795, espécie comumente citada para ecorregião NCCD por ser amplamente distribuída na Região Neotropical (Tyson Roberts, comunicação pessoal).

Gymnotus aff. carapo (Linnaeus, 1758), conhecido como sarapó ou carapó, tem dois lotes de espécimes da bacia do rio Jaguaribe depositados na coleção ictiológica da UFPB, um coletado no riacho do Sovaco, afluente do rio Timbó no bairro Cidade Universitária (UFPB 0344) e outro em local não especificado do rio Jaguaribe, a jusante da Mata do Buraquinho (UFPB 0345). Diversos autores (e.g. Campos-daPaz e Costa 1996; Albert e Crampton 2003) apontaram que G. carapo se trata de um grupo de espécies distribuídas em diferentes bacias do norte e leste da América do Sul. Albert e Crampton (2003) erroneamente indicaram que as espécies deste grupo não ocorrem no nordeste brasileiro ao leste da bacia do rio Parnaíba. Ademais, se equivocaram ao mapear a ocorrência de Gymnotus bahianus Campos-da-Paz e Costa (1996) nas bacias costeiras dos Estados de Pernambuco e Paraíba. Gymnotus bahianus distingue-se facilmente das espécies do grupo carapo por não apresentar as barras oblíquas nos lados do corpo que caracterizam o padrão de coloração destas últimas, e até o presente não é conhecido da ecorregião Nordeste Médio-Oriental (sensu Rosa et al. 2003; Campos-da-Paz \& de Santana 2019). Recentemente, uma nova espécie de Gymnotus do "clado Gymnotus carapo" foi descrita na Unidade de Conservação Refúgio Ecológico Charles Darwin, Pernambuco, Brasil. (Gymnotus darwini Campos-da-Paz \& de Santana 2019). Essa nova espécie é a primeira descrita para áreas costeiras na ecorregião Nordeste Médio-Oriental (Campos-da-Paz \& de Santana 2019), e provavelmente há registros desse táxon em outras drenagens costeiras nos estados de Pernambuco, Paraíba e Rio Grande do norte, como sugere Campos-da-Paz \& de Santana (2019). Portanto, recomenda-se a revisão sistemática do "grupo Gymnotus carapo" nessa ecorregião. 
O número de espécies de peixes registradas neste estudo, 21, demonstra que a bacia do rio Jaguaribe apresenta riqueza relativamente similar àquela registrada em outros rios costeiros sobre influência da Mata Atlântica da ecorregião Nordeste Médio-Oriental. Como citado anteriormente, Gomes-Filho (1999) realizou o inventário das espécies de peixes, apenas da ordem dos Characiformes, das bacias costeiras do Estado da Paraíba: Abiaí, Camaratuba, Gramame, Jaguaribe, Paraíba do Norte e Mamanguape. Nesse estudo foram registradas 18 espécies dentro de 13 gêneros e sete famílias. O trabalho de Gomes-Filho (1999) foi realizado com dados da coleção de peixes da UFPB. Torelli et al. (1997) e Gomes-Filho e Rosa (2001) realizaram novos inventários na bacia do rio Gramame, bacia também sobre domínio da Mata Atlântica, porém mais extensa que a bacia rio Jaguaribe. Na bacia do rio Gramame, Torelli et al. (1997) registraram 22 espécies distribuídas em 22 gêneros e 15 famílias e Gomes-Filho e Rosa (2001) registraram 32 espécies das quais 23 eram de água doce.

Rosa \& Groth (2004) realizaram o levantamento dos brejos de altitudes dos estados da Paraíba e Pernambuco. Os brejos de altitudes são considerados resquícios de Mata Atlântica em regiões de Caatinga, neste trabalho foram registradas 27 espécies pertencentes a 23 gêneros de 12 famílias. Na microbacia do rio Pratagi, Estado do Rio Grande do Norte, Paiva et al. (2014) listaram 22 espécies dentro de 18 famílias, destas, 13 eram de água doce. Deste modo, a diversidade total dos peixes do rio Jaguaribe, seria compatível com a pequena extensão desta bacia costeira, e comparável a dos rios Pratagi e Gramame. Em comparação com esta última bacia, a ictiofauna do rio Jaguaribe mostrou grande similaridade na sua composição em espécies de água doce, com apenas uma espécie (Compsura heterura) não compartilhada. A diferença no número total de espécies de água doce entre as duas bacias, 17 na do rio Jaguaribe e 22 na do rio Gramame, pode ser explicada pela maior extensão desta última.

Todavia, deve-se ressaltar que a bacia do rio Jaguaribe esteja passando por um processo de empobrecimento, com possíveis extinções locais de espécies, em função da degradação ambiental generalizada, particularmente aquela decorrente da poluição hídrica, supressão da mata ciliar e modificações da sua área de drenagem a partir da canalização de grande parte do curso principal do rio. A degradação ambiental da bacia é decorrente da ocupação urbana desordenada das cidades de João Pessoa e de Cabedelo e atinge praticamente toda a sua extensão. Esta degradação foi diagnóstica a partir das expedições dos anos 2017 e 2018 e inclui a contaminação hídrica por esgotos não tratados, a destruição da vegetação ciliar e a ocupação indevida das margens e às vezes do próprio leito dos rios. O trecho do rio Jaguaribe que corta a Mata do Buraquinho, embora historicamente modificado para a exploração do manancial no abastecimento urbano, é sem dúvida a porção menos impactada da bacia, particularmente pela presença da cobertura vegetal. 


\section{AGRADECIMENTOS}

A iniciativa deste trabalho partiu de Antônio Augusto de Sá Neto, que em 2005 completou sua monografia de graduação sobre o tema. Os autores agradecem a Fernando Groth, Erich de Freitas Mariano, Janaína da Fonseca Conde e Olívio Travassos Moura, pelo auxílio nas atividades de campo; ao Dr. Fernando Carvalho (UFMT) pela identificação dos Characidae e a Tyson Roberts (California Academy of Sciences) pelo exame dos Synbranchidae.

\section{REFERÊNCIAS}

Abell, R.; M. L. Thieme; C. Revenga; M. Bryer; M. Kottelat; N Bogutskaya; B. Coad; N. Mandrak; S. C. Balderas; W. Bussing; M. L. J. Stiassny; P Skelton; G. R. Allen; P. Unmack; A. Naseka; R. Ng; N. Sindorf; J. Robertson; E. Armijo; J. V. Higgins; T. J. Heibel; E. Wikramanayake; D. Olson; H. L. López; R. E. Reis; J. G. Lundberg; M. H. Sabaj-Pérez; P. Petry. 2008. Freshwater ecoregions of the world: a new map of biogeographic units for freshwater biodiversity conservation. BioScience, 58(5), 403-414.

Albert, J. S. \& W. G. R. Crampton. 2003. Seven new species of the Neotropical electric fish Gymnotus (Teleostei, Gymnotiformes) with a redescription of G. carapo (Linnaeus). Zootaxa 287: 1-54.

Barbosa, M. R. D. V. 1996. Estudo florístico e fitossociológico da Mata do Buraquinho, remanescente de Mata Atlântica em João Pessoa, PB.

BRASIL. 2014. Lista Nacional Oficial de Espécies da Fauna Ameaçada de Extinção Portaria $\mathrm{N}^{\circ}$ 443, de 17 de dezembro de 2014. Ministério do Meio Ambiente. Diário Oficial da União, Brasília, Seção 1(245): 121-130.

Britski, H. A.; Sato, Y. \& A. B. S. Rosa. 1984. Manual de identificação de peixes da região de Três Marias, com chaves de identificação para os peixes da bacia do Rio São Francisco. CODEVASF, Brasília, 143p.

Buckup, P. A. 1998. Biodiversidade dos peixes da mata atlântica. URL: http://www.bdt. fat.org.br/workshop/mata.atlantica/SE-S/peixes\#buckup. Data de acesso: 16/04/2005.

Buckup, P. A., N. A. Menezes, \& M. S. A. Ghazzi. 2007. Catálogo das espécies de peixes de água doce do Brasil (Vol. 1). Rio de Janeiro: Museu Nacional. 
Burger, R.; A. M. Zanata \& P. Camelier. 2011. Estudo taxonômico da ictiofauna de água doce da bacia hidrográfica do Recôncavo Sul, Bahia, Brasil. Biota Neotropica (Edição em português. Impresso), v. 11, p. 273-290.

Camelier, P. \& A. M. Zanata. 2014. Biogeography of freshwater fishes from the Northeastern Mata Atlântica freshwater ecoregion: distribution, endemism, and area relationships. Neotropical Ichthyology (Impresso), v. 12, p. 683-698.

Campos-da Paz \& W. J. E. M. Costa. 1996. Gymnotus bahianus sp. nov., a new gymnotid fish from eastern Brazil (Teleostei: Ostariophysi: Gymnotiformes), with evidence for the monophyly of the genus. Copeia 1996(4): 937-944.

Campos-da-Paz, R., \& C. D. de Santana. 2019 A New Species of the Electric Knifefish Gymnotus Linnaeus (Gymnotiformes: Gymnotidae) from Northeastern Brazil. Copeia, $107(1), 144^{-151 .}$

Costa, W.J.E.M.; T. P. A. Ramos; L.C. Alexandre; R. T. C. Ramos. 2010. Cynolebias parnaibensis, a new seasonal killifish from the Caatinga, Parnaíba River basin, northeastern Brazil, with notes on sound producing courtship behavior (Cyprinodontiformes: Rivulidae). Neotropical Ichthyology (Impresso), v. 8, p. 283288.

Fricke, R., W. N. Eschmeyer \& R. Van der Laan, (eds) 2019. Eschmeyer's Catalog of Fishes: Genera, Species, References. (http://researcharchive.calacademy.org/research/ ichthyology/catalog/fishcatmain.asp). Versão eletrônica acessada em 19 Abril 2019.

Géry, J. 1977. Characoids of the world. T.F.H Publications, Nepture City, U.S.A. 672p.

Gomes-Filho, G. 1999. Characiformes (Actinopterygii: Ostariophysi) das bacias costeiras do Estado da Paraíba. Dissertação de Mestrado, Universidade Federal da Paraíba, João Pessoa.

Gomes-Filho, G. \& R. S. Rosa. 2009. Inventário da ictiofauna da bacia do rio Gramame - PB, Brasil. Pp. 167-175 In: Barbosa, JEL, Watanabe, T \& Paz, RJ. (orgs.). A bacia do Rio Gramame: biodiversidade, uso e conservação. Campina Grande: EdUEPB, 229p.

Gosse, J. P. 1975. Révision du genre Geophagus (Pisces Cichlidae). Academie royale des Sciences d'Outre-Mer, Bruxelles, 172p.

Ihering, R. V. 1931. Cyprinodontes Brasileiros (Peixes "Guarús"). Systematica e informações biológicas. I. Archivos do Instituto Biológico de São Paulo, 4: 243-28o. 
Kullander, S. O. 1983. A revision of the South American cichlid genus Cichlasoma (Teleostei: Cichlidae). Naturhistoriska Riksmuseet, Stockholm, 296p.

Langeani, F., P.A. Buckup; L.R. Malabarba; L.H.R. Py-Daniel; C.A.S. Lucena; R.S. Rosa; J.A.S. Zuanon; Z.M.S. Lucena; M.R. De Britto; O.T. Oyakawa \& G. Gomes-Filho. 2009. Peixes de Água Doce. Em Estado da arte e perspectivas para a zoologia no Brasil (R.M. Rocha \& W.A.P. Boeger, orgs.). Ed. UFPR, Curitiba, p.211-230.

Lima S. M. Q.; T. P. A. Ramos; M. J. Silva; R. S. Rosa. 2017. Diversity, Distribution, and Conservation of the Caatinga Fishes: Advances and Challenges. In: Silva JMC, Leal IR, Tabarelli M. (Orgs) Caatinga. Springer. p.97-131.

Malabarba, L. R. \& R. E. Reis. 1987. Manual de técnicas para a preparação de coleções zoológicas. 36. Peixes. Sociedade Brasileira de Zoologia, Campinas, 14p.

Melo, A. S. T; E. L. Alves; J. L. Rodriguez; M. A. Tavares; M. A. C. Dantas; M. M. M. Guimarães; R. L. P. Gomes \& W. D. Heckendorff. 2001. Os aglomerados subnormais dos vales do Jaguaribe e Timbó: Análise geo-ambiental e qualidade do meio ambiente. Relatório de Pesquisa. Centro Universitário de João Pessoa - Unipê, João Pessoa, 132p.

Menezes, N.A., S.H. Weitzman, O.T. Oyakawa, F.C.T. Lima, R.M.C. Castro \& M.J. Weitzman. 2007. Peixes de água doce da Mata Atlântica: lista preliminar das espécies e comentários sobre conservação de peixes de água doce neotropicais. São Paulo: Museu de Zoologia da Universidade de São Paulo. 407 pp.

Paiva, M. P. 1995. A fauna nordestina. Pp. 87-141 in: Paiva, M. P. \& E. Campos (eds.). Fauna do nordeste do Brasil: conhecimento científico e popular. Editora Banco do Nordeste do Brasil, Fortaleza.

Paiva, R.E.C., S.M.Q. Lima; T.P.A. Ramos \& L.F. Mendes. 2014. Fish fauna of Pratagi river coastal microbasin, extreme north Atlantic Forest, Rio Grande do Norte State, northeastern Brazil. Check List 10(5): 968-975.

Ramos, R. T. C.; T. P. A. Ramos; R. S. Rosa; G. B. M. Beltrão \&, F. Groth. 2005. Diversidade de Peixes (Ictiofauna) da bacia do rio Curimataú, Paraíba. In Análise das Variações da Biodiversidade do Bioma Caatinga: Suporte das Estratégias Regionais de Conservação (F.S. Araujo, M.J.N. Rodal \& M.R.V. Barbosa, eds.), Ministério do Meio Ambiente, Brasília, p.291-318.

Ramos, T. P. A.; L. F. Barros-Neto; H. A. Britski; \& S. M. Q. Lima. 2013. Parotocinclus seridoensis, a new hypoptopomatine catfish (Siluriformes: Loricariidae) from the upper rio Piranhas-Açu basin, northeastern Brazil. Neotropical Ichthyology, 11(4), 787-796. 
Ramos, T. P. A.; R. T. C. Ramos \& S. A. Q. A. Ramos. 2014. Ichthyofauna of the Parnaíba river Basin, Northeastern Brazil. Biota Neotropica 14(3):1-6.

Ramos, T. P. A.; J. A. S. Lima; S. Y. L. Costa; M. J. Silva; R. C. Avellar; \&, L. OliveiraSilva. 2018. Continental ichthyofauna from the Paraíba do Norte River basin pretransposition of the São Francisco River, Northeastern Brazil. Biota Neotropica, 18(4). https://dx.doi.org/10.1590/1676-o611-bn-2017-0471.

Reis, R. E, 1992. Serão suficientes os sistematas de peixes neotropicais? In: Agostinho, A. A. \& E. Benedito-Cecilio. (eds). 1992. Situação atual e perspectivas da ictiologia no Brasil. Documentos do IX Encontro Brasileiro de Ictiologia. Universidade Estadual de Maringá, 127p.

Reis, R. E. 1993. Filogenia da família Callichthyidae (Ostariophysi, Siluriformes), com uma revisão taxonômica do gênero Hoplosternum. Tese de Doutorado, Universidade de São Paulo, São Paulo.

Reis, R. E.; S. O. Kullander \& C. J. Ferraris-Jr. 2003. Checklist of the freshwater fishes of South and Central America. Editora da Pontifícia Universidade Católica do Rio Grande do Sul, Porto Alegre, 742p.

Reis, R. E., Albert, J. S., Di Dario, F., Mincarone, M. M., Petry, P., \& Rocha, L. A. 2016. Fish biodiversity and conservation in South America. Journal of Fish.

Rosa, R. S, Menezes, N. A, Britski H. A; W. J. E. M Costa, \& F. Groth. 2003. Diversidade, Padrões de Distribuição e Conservação dos Peixes da Caatinga. Pp. 135-18o in: Leal, I.R., M. Tabarelli \& J. M. C. Silva (eds.). Ecologia e Conservação da Caatinga. Editora Universitária da UFPE, Recife.

Rosa, R. S. \& F. Groth. 2004. Ictiofauna dos ecossistemas de brejos de altitude de Pernambuco e Paraíba. Pp. 201-210 in: Porto, K. C; J. J. P. Cabral \& M. Tabarelli (orgs.). Brejos de altitude em Pernambuco e Paraíba: história natural, ecologia e conservação. Ministério do Meio Ambiente, Brasília.

ROSA, P. R. O. Agonia de um rio Marcado para morrer.João Pessoa:Jornal Contraponto, 23 a 29 março de 2009, caderno A, p. 5.

Sarmento-Soares, L. M.; H. A. Britski; M. Anjos; A. M. Zanata; R. F. Martins-Pinheiro \& M. G Barretto. 2016. First record of genus Imparfinis to a coastal Brazilian river basin: I. borodini Mees and Cala in rio de Contas, Bahia. Check List (São Paulo. Online), v. 12, p. 1-7. 
Silva, M. J. D.; T. P. A. Ramos; V. D. Diniz; R. T. D. C. Ramos \&, E. S. F. Medeiros. 2014. Ichthyofauna of Seridó/Borborema: a semi-arid region of Brazil. Biota Neotropica, 14(3).

da Silva, M. J.; B. G. Costa; T. P. A. Ramos; P. Auricchio; \& S. M. Q. Lima. 2015. Ichthyofauna of the Gurgueia river, Parnaíba river basin, northeastern Brazil. Check List, $11(5), 1765$.

Torelli, J; I. L. Rosa \& T. Watanabe. 1997. Ictiofauna do rio Gramame, Paraíba, Brasil. Iheringia ser. zool. 82: 67-73.

Vari, R. P.; A. M. Zanata; P. Camelier. 2010. New species of Cyphocharax (Ostariophysi: Characiformes: Curimatidae) from the Rio de Contas drainage, Bahia, Brazil. Copeia, v. 2010, p. 382-387.

Zanata, A. M.; B. S. Bezerra; C. Zawadzki. 2013. A new dark-dotted species of Hypostomus Lacépède (Siluriformes: Loricariidae) from rio Paraguaçu, Bahia State, Brazil. Neotropical Ichthyology (Impresso), v. 11, p. 247-256.

Zanata, A. M. \& Serra, J. P. 2010. Hasemania piatan, a new characid species (Characiformes: Characidae) from headwaters of rio de Contas, Bahia, Brazil. Neotropical Ichthyology (Impresso), v. 8, p. 21-26.

\section{MATERIAL EXAMINADO}

Astyanax bimaculatus - UFPB 0384 - Canal artificial na Mata do Buraquinho, rio Jaguaribe, João Pessoa, PB (4); UFPB o462 - rio Jaguaribe, João Pessoa, PB (3); UFPB o684 - rio Jaguaribe, João Pessoa, PB (1); UFPB o699 - rio Jaguaribe, João Pessoa, PB (2); UFPB o8o8 - rio Jaguaribe, João Pessoa, PB (8); UFPB o812 - rio Jaguaribe, João Pessoa, PB (2); UFPB o846 - rio Jaguaribe, João Pessoa, PB (2); UFPB 5972 - rio

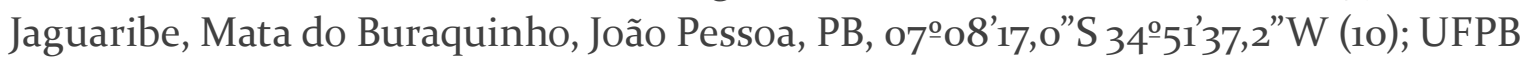
6029 - rio Jaguaribe, próximo à Mata do Buraquinho, João Pessoa, PB (4). Astyanax fasciatus - UFPB 0475 - Alagados do Bessa, João Pessoa, PB (16). Cichlasoma orientale UFPB o520 - rio Jaguaribe, João Pessoa, PB (2); UFPB o8o4 - rio Jaguaribe, João Pessoa, PB (2); UFPB o843 - rio Jaguaribe, João Pessoa PB (1); UFPB o961 - rio Jaguaribe, João Pessoa, PB (1); UFPB 1125 - rio Jaguaribe, João Pessoa, PB (2); UFPB 1334 - rio Jaguaribe, Mata do Buraquinho, João Pessoa, PB (6); UFPB 1336 - rio Jaguaribe, próximo à Mata do Buraquinho, João Pessoa, PB (1); UFPB 1337 - rio Jaguaribe, próximo à Mata do Buraquinho (1); UFPB 1373 - rio Jaguaribe, Mata do Buraquinho, João Pessoa, PB (1); UFPB 1380 - rio Jaguaribe, próximo à Mata do Buraquinho, João Pessoa, PB (3); UFPB 1397 - rio Jaguaribe, João Pessoa, PB (2); UFPB 5971 - rio Jaguaribe, Mata do 
Buraquinho, João Pessoa, PB (3); UFPB 5971 - rio Jaguaribe, Mata do Buraquinho,

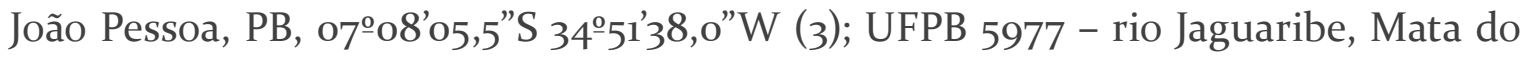
Buraquinho, João Pessoa, PB, o7º08'10,5”S 34ํ5139,7”W (1); UFPB 5980 - rio Jaguaribe,

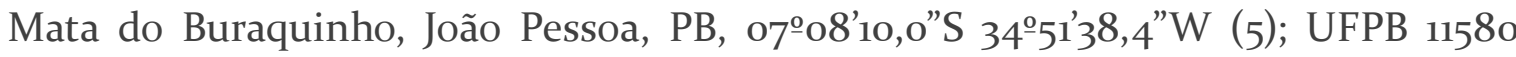
- rio Jaguaribe, João Pessoa, PB, 0705'02,9”S 034 50’03,9”W. Compsura heterura - UFPB 5973 - rio Jaguaribe, Mata do Buraquinho, João Pessoa, PB, o7º08'17,o"S 34ํำ'37,2"W (10); UFPB 5979 - rio Jaguaribe, Mata do Buraquinho, João Pessoa, PB, o7º8'13,6”S 34ํำ'49,7”W (5); UFPB 5983 - rio Jaguaribe, prox. à Mata do Buraquinho, João Pessoa, PB (11). Dormitator maculatus - UFPB 0370 - Canal artificial na Mata do Buraquinho, rio Jaguaribe, João Pessoa, PB (3); UFPB 0427 - Canal artificial na Mata do Buraquinho, rio Jaguaribe, João Pessoa, PB (4); UFPB o696 - rio Jaguaribe, João Pessoa, PB (15); UFPB o807 - rio Jaguaribe, João Pessoa, PB (2); UFPB o848 rio Jaguaribe, João Pessoa, PB (17); UFPB o849 - rio Jaguaribe, João Pessoa, PB (14); UFPB 1345 - rio Jaguaribe, Mata do Buraquinho, João Pessoa, PB (1); UFPB 1346 - rio Jaguaribe, Mata do Buraquinho, João Pessoa, PB (1); UFPB 1347 - rio Jaguaribe, Mata do Buraquinho, João Pessoa, PB (1). Geophagus brasiliensis - UFPB 0378 - rio Jaguaribe, PB (2); UFPB o38o - rio Jaguaribe, PB (3); UFPB o381 - rio Jaguaribe, PB (2); UFPB 0497 - rio Jaguaribe, PB (3); UFPB 0523 - Canal artificial na Mata do Buraquinho, rio Jaguaribe, João Pessoa, PB (2); UFPB 0524 - Canal artificial na Mata do Buraquinho, rio Jaguaribe, João Pessoa, PB (1); UFPB o695 - rio Jaguaribe, João Pessoa, PB (1); UFPB o805 - rio Jaguaribe, João Pessoa, PB (1); UFPB o81o - rio Jaguaribe, João Pessoa, PB (1); UFPB o811 - rio Jaguaribe, João Pessoa, PB (1); UFPB 1333 - rio Jaguaribe, Mata do Buraquinho, João Pessoa, PB (XX); UFPB 1372 - rio Jaguaribe, Mata do Buraquinho, João Pessoa, PB (2); UFPB 1776 - rio Jaguaribe, João Pessoa, PB (10); UFPB 1778 rio Jaguaribe, João Pessoa, PB (6); UFPB 2031 - rio Jaguaribe, João Pessoa, PB (1); UFPB 2169 - rio Jaguaribe, João Pessoa, PB (3); UFPB 2170 - rio Jaguaribe, João Pessoa, PB (1); UFPB 5975 - rio Jaguaribe, Mata do Buraquinho, João Pessoa, PB,

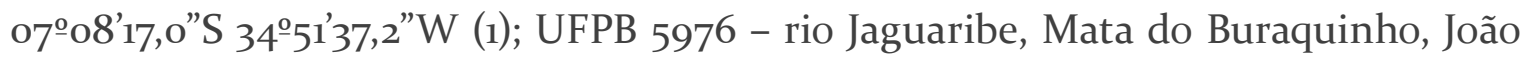
Pessoa, PB, 0708'10,5”S 34ํำ39,7”W (1); UFPB 6032 - rio Jaguaribe, próximo à Mata do Buraquinho, João Pessoa, PB (7). Gymnotus cf. carapo - UFPB 0344 - Riacho do Sovaco ou Timbó, próximo à Cidade Universitária, João Pessoa, PB (2); UFPB 0345 - rio Jaguaribe, João Pessoa, PB (1). Hemigrammus cf. unilineatus - UFPB 3632 - rio Jaguaribe, Mata do Buraquinho, João Pessoa, PB (18). Hemigrammus marginatus UFPB 2168 - rio Jaguaribe, Mata do Buraquinho, João Pessoa, PB (19). Hoplerythrinus unitaeniatus - UFPB 0350 - Canal artificial na Mata do Buraquinho, rio Jaguaribe, João Pessoa, PB (3). Hoplias malabaricus - UFPB ooo4 - rio Jaguaribe, João Pessoa, PB (1); UFPB 0359 - rio Jaguaribe, João Pessoa, PB (1); UFPB o716 - rio Jaguaribe, João Pessoa, PB; UFPB 3602 - rio Jaguaribe, João Pessoa, PB (1); UFPB 5970 - rio

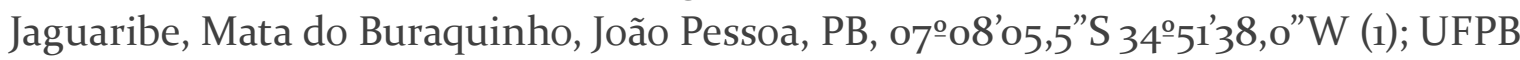
6028 - rio Jaguaribe, próximo à Mata do Buraquinho, João Pessoa, PB (2). Megalechis personata - UFPB o051 - rio Jaguaribe, BR-230, João Pessoa, PB (1); UFPB 0052 - rio Jaguaribe, BR-230, Água Represada (1); UFPB 3630 - Lagunas do Intermares, bacia do rio Jaguaribe, Cabedelo, PB (1). Oreochromis niloticus - UFPB 5978 - rio Jaguaribe, 


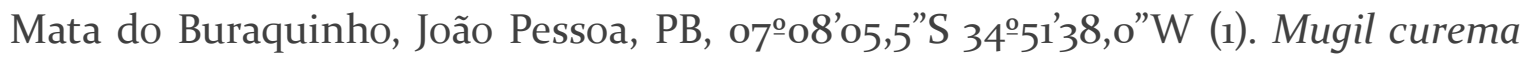

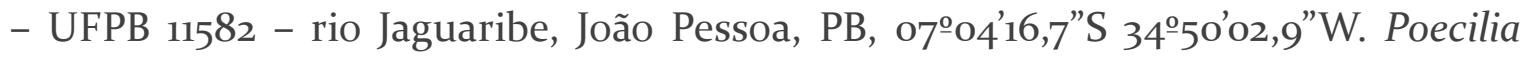
reticulata - UFPB 0522 - Canal artificial na Mata do Buraquinho, rio Jaguaribe, João Pessoa, PB (68); UFPB 1341 - rio Jaguaribe, próximo à Mata do Buraquinho, João Pessoa, PB (29); UFPB 5974 - rio Jaguaribe, Mata do Buraquinho, João Pessoa, PB,

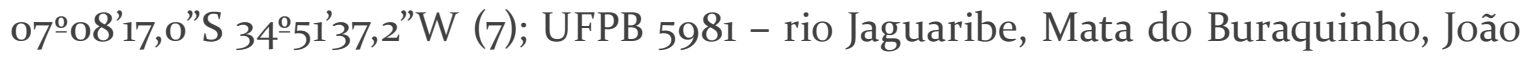

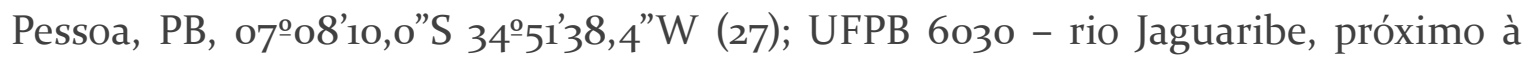
Mata do Buraquinho, João Pessoa, PB (1); UFPB 11574 - rio Jaguaribe, João Pessoa, $\mathrm{PB}, 07^{\circ} 05^{20}$, $4^{\prime \prime} \mathrm{S}$ 034 50'44,7”W. Poecilia vivipara - UFPB 0005 - BR-230, rio Jaguaribe, João Pessoa, PB (2); UFPB 0521 - Canal artificial na Mata do Buraquinho, rio Jaguaribe, João Pessoa, PB (30); UFPB o845 - rio Jaguaribe João Pessoa, PB (3); UFPB 1391 - rio Jaguaribe, próximo à Mata do Buraquinho, João Pessoa, PB (186); UFPB 6031 - rio Jaguaribe, próximo à Mata do Buraquinho, João Pessoa, PB (4); UFPB 11575 - rio Jaguaribe, João Pessoa, PB, o705’16,3”S 034 ${ }^{\circ} 50^{\prime} 49,6$ ”'W. Steindachnerina notonota - UFPB 1383 - rio Timbó, imediações da favela dos Bancários, João Pessoa, PB (2). Synbranchus sp. - UFPB 0525 - Canal artificial na Mata do Buraquinho, rio Jaguaribe, João Pessoa, PB (1); UFPB o691 - rio Jaguaribe, João Pessoa, PB (1); UFPB 1322 - rio Jaguaribe, próximo à Mata do Buraquinho, João Pessoa, PB (3); UFPB 1773 - rio Jaguaribe, Mata do Buraquinho, João Pessoa, PB (1); UFPB 1992 - rio Jaguaribe, Mata do Buraquinho, João Pessoa, PB (1); UFPB 5981 - rio Jaguaribe, Mata do Buraquinho,

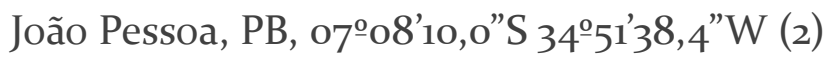

